\title{
Investigation of Temperature Fluctuation of Diode-Rectified Multiphase AC Arc by High-Speed Visualization
}

\author{
Manabu TANAKA ${ }^{1}$, Yuta KugimiYA ${ }^{1}$, Hiroki MaruYAmA ${ }^{1}$, Takayuki WATANABE ${ }^{1}$, and Tsugio MATSUURA $^{2}$ \\ ${ }^{1}$ Faculty of Engineering, Kyushu University, 744 Motooka, Fukuoka-shi, Fukuoka 819-0395, Japan \\ ${ }^{2}$ Taso Arc Co. Ltd., 10-11 Imai, Ohno-shi, Fukui 912-0424, Japan
}

\begin{abstract}
An innovative multiphase $A C$ arc was drastically improved by diode-rectification technique with bipolar electrode. Temperature fields and arc behaviour of the diode-rectified multiphase AC was successfully visualized on the basis of the high-speed camera technique with appropriate band-pass filter optics. Arc temperature was measured by Boltzmann plot method with two line emissions from atomic argon at $675.2834 \mathrm{~nm}$ and $794.8176 \mathrm{~nm}$. Arc temperature fluctuates in the range from 7,000 to $13,000 \mathrm{~K}$. The arc temperature near the cathode was higher than $13,000 \mathrm{~K}$, while that near the anode was about 10,000 K. Arc temperature in the centre region in the furnace was about 7,000-9,000 K, which is sufficiently high to melt and evaporate the refractory metals or ceramics. Obtained results suggested the diode-rectified multiphase AC arc is a promising thermal plasma source for material processing at high productivity.
\end{abstract}

\section{Introduction}

A multiphase $\mathrm{AC}$ arc (MPA) is one of the most attractive thermal plasma sources due to its advantages such as higher energy efficiency compared with conventional thermal plasmas. Therefore, the MPA has been applied to an in-flight glass melting technology (Watanabe et al., 2010). Furthermore, it is expected to be utilized in nanomaterial fabrication processes owing to its high processing rate. Fundamental studies for practical use of MPA in industry have been intensively reported in terms of the arc stability (Tanaka et al., 2011), the temporal and spatial characteristics of the arc discharge (Liu et al., 2013), arc temperature fluctuation (Okuma et al., 2018, 2019), and the electrode phenomena (Tanaka et al., 2013, 2016, Hashizume et al., 2015).

Electrode erosion in $\mathrm{AC}$ arc is one of the most important issues to be solved because it determines the electrode lifetime and purity of the products. Appropriate properties for cathode and anode in arc discharge are different. Low work function and high melting point are important cathode properties for stable electron emission. Contrastingly, high thermal conductivity is required for anode as electron recipient. However, there is a lack of appropriate electrode material which satisfies required properties at both cathodic and anodic periods. Tungsten based electrode are commonly used as AC electrode for stable thermionic emission, although the thermal conductivity is insufficient. This fact led to severe erosion in conventional single-phase AC arc (Hinata et al., 1993) and MPA (Hashizume et al., 2015).

Diode-rectified MPA (DRMPA) has been successfully developed to solve the above issue (Tanaka et al., 2017). Diode rectification technique was utilized to separate an AC electrode into a pair of cathode and anode. This separation of the AC electrode led to drastic improvement of electrode erosion. Cathodic erosion was reduced by the absence of the anodic heat transfer in the DRMPA. Furthermore, erosion at anodic period was also drastically suppressed because of the high thermal conductivity of the copper anode in the DRMPA. Understanding of fundamental phenomena in the DRMPA has been poorly understood because of its novelty in spite of its importance. In particular, the spatiotemporal characteristics of the arc temperature are essential to utilize the DRMPA in industrial field.

The purpose of the present work is to investigate spatiotemporal characteristics of the arc temperature field. Visualization technique by a high-speed camera with appropriate band-pass filters was applied.

\section{Experimental Setup}

Schematic electric circuits and conceptual waveform of arc current are shown in Figures 1 and 2. Twelve diodes are placed between the electrodes and transformers for DRMPA. The electrodes were divided into pairs of cathode and anode, namely bipolar electrodes. Each electrode consists of cathode and anode. The cathode was made of $2 \mathrm{wt} \%$-thoriated tungsten with $3.2 \mathrm{~mm}$ in diameter. The anode was made of copper rod with $25 \mathrm{~mm}$ in diameter and was directly cooled by city water.

Figures 3 and 4 show the schematics of the experimental setup with measurement system and the electrode configurations. Six pairs of the electrodes were symmetrically arranged at the angles of $60 \mathrm{deg}$. Odd

* Corresponding author: watanabe@chem-eng.kyushu-u.ac.jp 
numbered cathodes were placed above the corresponding anodes, while even numbered anodes were placed above the cathodes. DRMPA was generated among 6 bipolar electrodes in the chamber which was filled by argon under atmospheric pressure. The gap distance between opposite electrodes, e.g. No1 and 4, was adjusted at $35 \mathrm{~mm}$. (a)

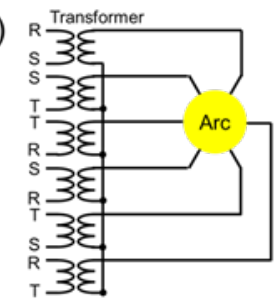

(b)

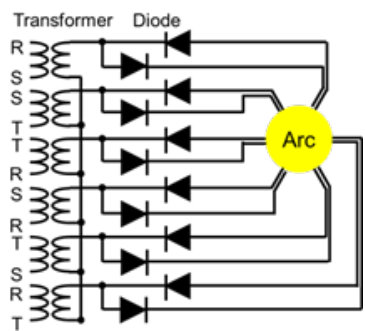

Figure 1. Schematic of the electric circuits in (a) the multiphase AC arc and (b) diode-rectified multiphase $\mathrm{AC}$ arc

(a)

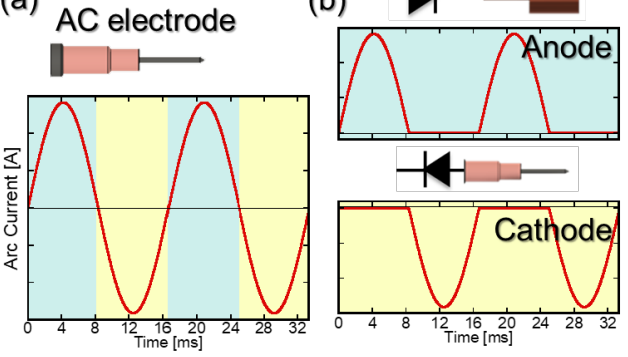

Figure 2. Conceptual waveform of arc current in (a) the multiphase $\mathrm{AC}$ arc and (b) diode-rectified multiphase $\mathrm{AC}$ arc

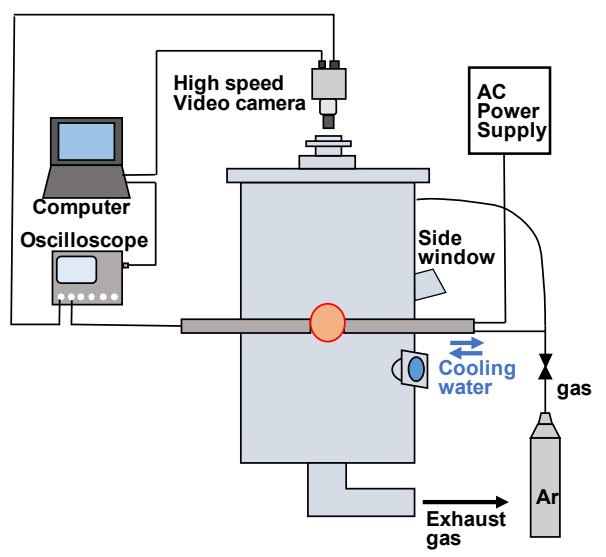

Figure 3. Schematic of arc furnace and measurement system

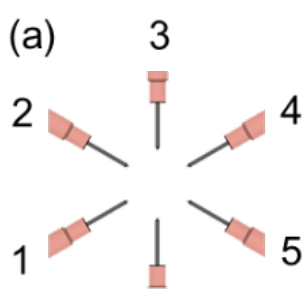

6 (b)

3

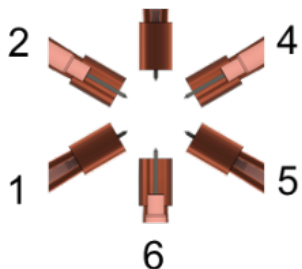

Figure 4. Electrode configuration of (a) the multiphase $\mathrm{AC}$ arc and (b) the diode-rectified multiphase $\mathrm{AC}$ arc
Electrode temperature during discharge was measured by the high-speed camera. Conventionally, electrode temperature measurement during the arc discharge was difficult due to the strong emissions from the arc. Recently developed technique with high-speed camera (FASTCAM SA5, Photron Ltd., Japan) was utilized in the present work. Only thermal radiation from the electrode surface was visualized without strong emissions from the arc by appropriate band-pass filters which transmission wavelengths were $785 \pm 2.5$ and $880 \pm 5 \mathrm{~nm}$. Then, surface temperature was measured on the basis of the twocolour pyrometry. Typical framerate was $1 \times 10^{4} \mathrm{fps}$ with shutter speed of 20-50 $\mu$ s.

Arc temperature in the DRMPA was also measured by the same high-speed camera mentioned above. The difference from the electrode temperature measurements was the observed wavelengths. The band-pass filters of $675 \pm 5 \mathrm{~nm}$ and $794 \pm 5 \mathrm{~nm}$, which include line emissions from atomic argon at $675.2834 \mathrm{~nm}$ and $794.8176 \mathrm{~nm}$ respectively were used. Excitation temperature of atomic argon was then calculated based on the Boltzmann plot method.

\section{Results and Discussions}

Arc behaviour in DRMPA was observed by high-speed camera system without band-pass filters. Figures 5 and 6 shows the high-speed snapshots of the MPA and the DRMPA during an AC cycle. Electrode No. 1 was in the anodic period from $0.00 \mathrm{~ms}$ to $8.33 \mathrm{~ms}$, while electrode No. 4 was in the cathodic period at the same time. In the case of MPA, strong cathode jet was observed at near the electrode No. 1 when the time was 4 ms. The strong anode jet near the electrode No. 4 was also observed at the same time. These arcs are connected around the canter region among the electrodes.

In the case of DRMPA, the strong cathode jet was observed at around the cathode No. 4 as well as the MPA case. On the other hand, multiple anode spots with weak anode jet were clearly observed from anode No. 1 at $4 \mathrm{~ms}$. This clear difference of the anodic arc behaviour between the MPA and the DRMPA can be explained by the effect of the metal vapour. High-speed camera observation with appropriate band-pass filter revealed that the tungsten vapour was observed at the anodic period of the MPA, while copper vapour was not observed in the case of the DRMPA. Therefore, this absence of the metal vapour in front of the anode in the DRMPA leads to the weaker anode jet.

Figures 7 and 8 shows the visualized temperature fields of the MPA and the DRMPA. These temperature distributions correspond to the high-speed snapshots in Figures 5 and 6 . The results indicated that the temperature of the both MPA and DRMPA were fluctuated in the range of $7,000 \mathrm{~K}$ to $13,000 \mathrm{~K}$. The arc temperature near the electrode was higher than $10,000 \mathrm{~K}$, while the temperature in the centre region in the furnace is lower than the $10,000 \mathrm{~K}$. The temperature in the centre region is important because most of the treated powders are injected into this region. 


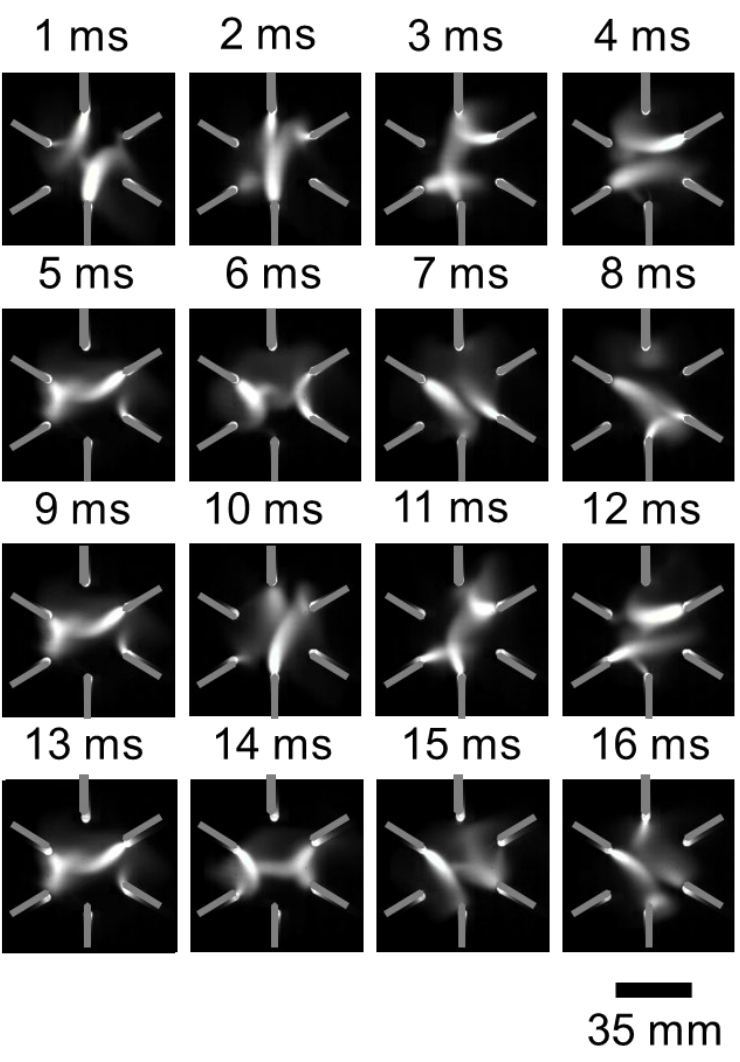

Figure 5. High-speed snapshots of the multiphase AC arc during one AC period. Grey part indicates the electrodes

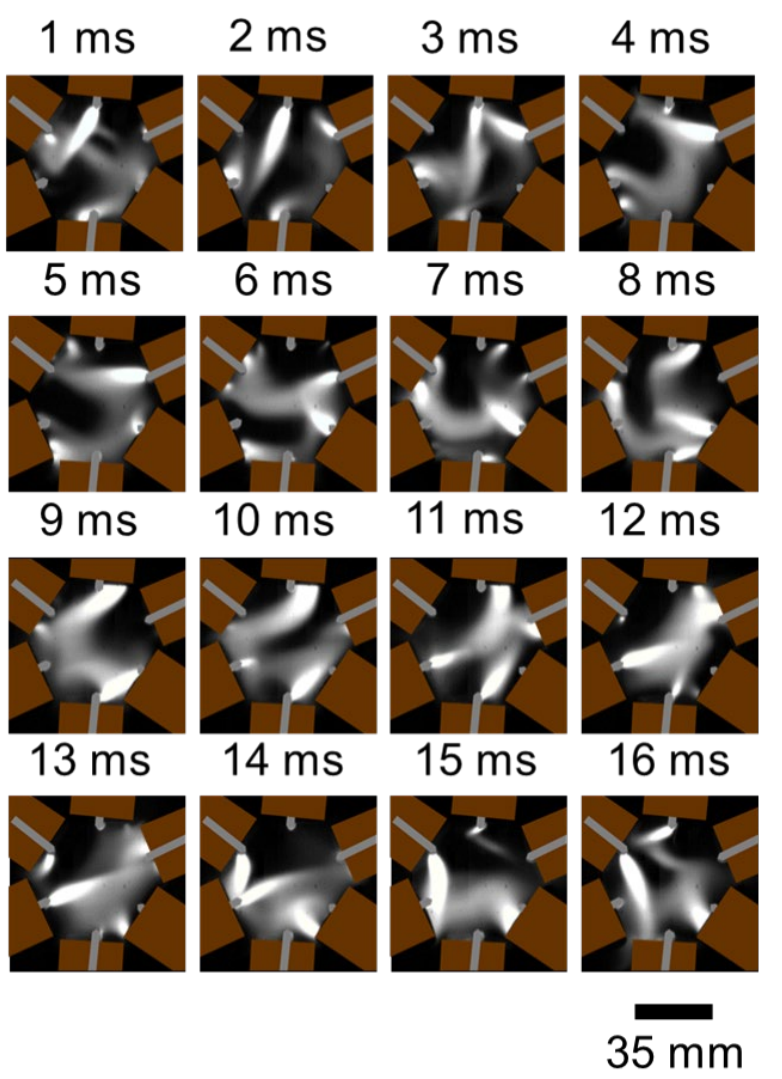

Figure 6. High-speed snapshots of the diode-rectified multiphase AC arc during one AC period. Grey and brown part indicate the cathode and anode, respectively

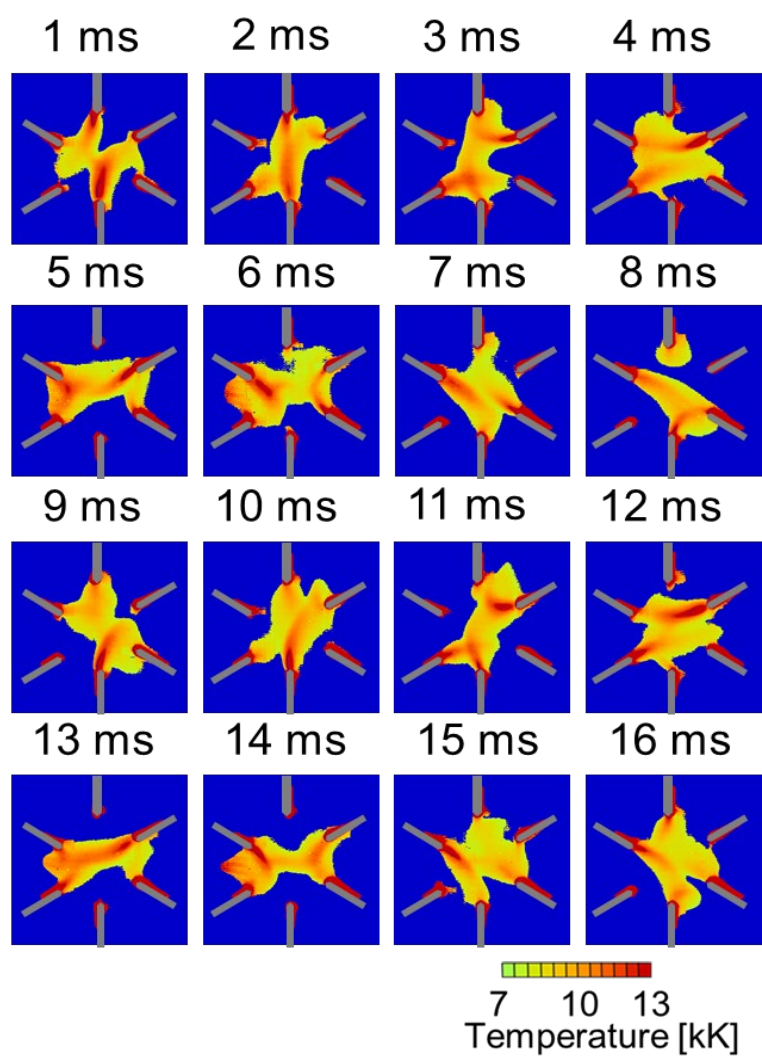

Figure 7. Arc temperature distributions of the multiphase AC arc during one AC period. Grey part indicates the electrodes

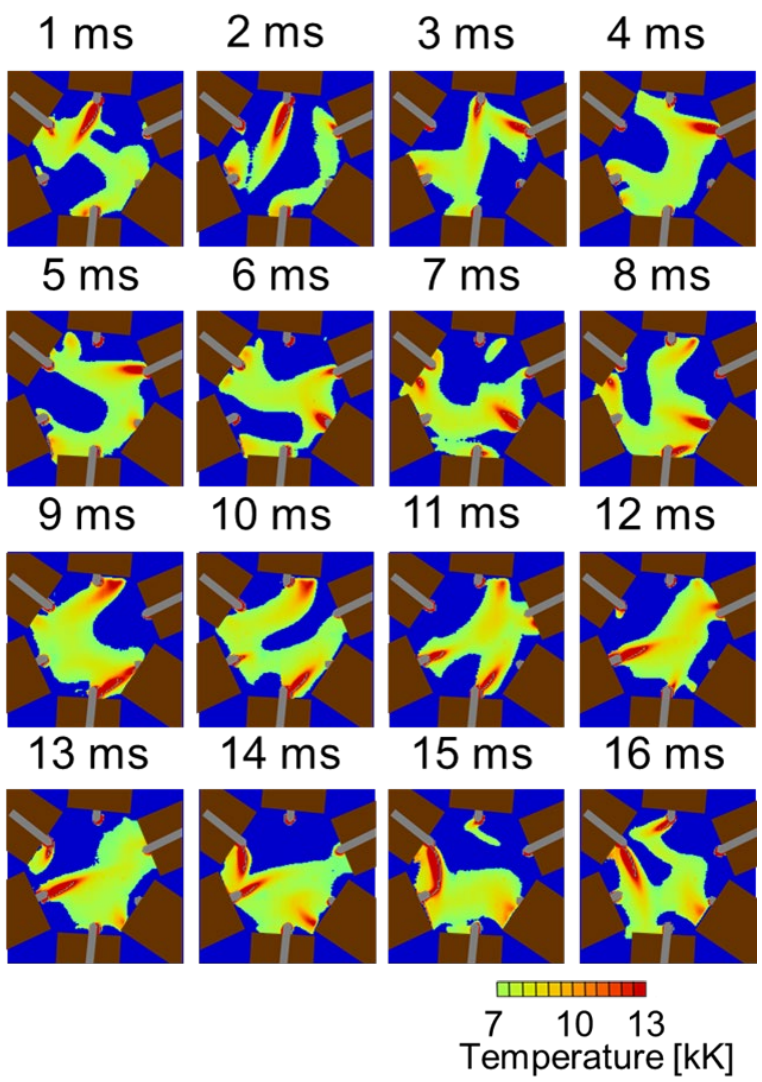

Figure 8. Arc temperature distributions of the diode-rectified multiphase $\mathrm{AC}$ arc during one AC period. Grey part and brown part indicate the cathode and anode, respectively 
Figure 9 presents the temperature fluctuation in the centre region of the furnace, which was estimated from the obtained temperature fields. Results indicated that the arc temperature in the DRMPA was lower than that in the MPA. The reason for this result is still unclear, but the relationship between the cathode jet and anode jet flow must have a strong effect on the temperature fluctuation. Arc temperature near the anode in the DRMPA was clearly lower than that near the cathode in the DRMPA and MPA. Moreover, the multiple anode spots can be observed in the DRMPA. These facts lead to the broadening of the arc region in the DRMPA, resulting in the relatively lower temperature in the DRMPA than that in the MPA.
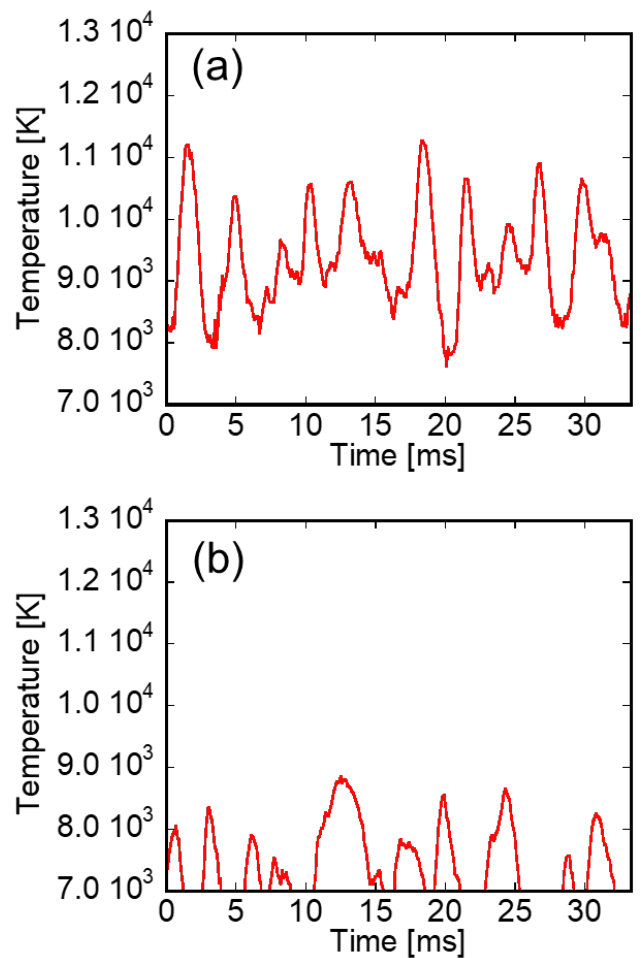

Figure 9. Temperature fluctuation at the centre of the furnace for (a) the multiphase AC arc, (b) the diode-rectified multiphase $\mathrm{AC}$ arc

\section{Conclusions}

Arc temperature field in the diode-rectified multiphase $\mathrm{AC}$ arc has been successfully visualized on the basis of the high-speed camera system with appropriate band-pass filters. Strong cathode jet and anode jet were observed in the multiphase AC arc, while strong cathode jet accompanies with the weak anode jet in the dioderectified multiphase $\mathrm{AC}$ arc. These different electrode jet behaviour leads to different spatial and temporal characteristics of the arc. Obtained results suggested that the diode-rectified multiphase AC arc is expected to be utilized in massive powder processing such as nanofabrication processes at high-productivity for a long time operation.

\section{Acknowledgements}

This work was partly supported by JSPS KAKENHI Grant Number 19K03808.

\section{References}

Hashizume, T., M. Tanaka, and T. Watanabe; "Investigation of Droplet Ejection Mechanism from Electrode in Multi-Phase AC Arc," Quart. J. Jpn. Weld. Soc., 33, 44s-48s (2015)

Hinata, T., K. Yasuda, and T. Onzawa, "Influence of Gastungsten-arc Electrode Material on AC TIG Arc Welding," Trans. Jpn. Weld. Soc., 24, 15-22 (1993)

Liu, Y., M. Tanaka, T. Ikeba, S. Choi, and T. Watanabe; "Fluctuation Measurement of Multi-Phase AC Arc and In-Flight Particle Temperature," J. Chem. Eng. Japan., 46, 672-676 (2013)

Okuma, T., T. Imatsuji, T. Hashizume, M. Tanaka, T. Watanabe, H. Nagai, and T. Koiwasaki; "Effects of Working Pressure on Temperature Profiles in Multi-Phase Ac Arc," J. Fluid Sci. Technol., 13, JFST0024 (2018)

Okuma, T., H. Maruyama, T. Hashizume, M. Tanaka, T. Watanabe, H. Nagai, and T. Koiwasaki; "Effects of Driving Frequency on Temperature in a MultiPhase AC Arc," IEEE Trans. Plasma Sci., 47, 32-38 (2019)

Tanaka, M., Y. Tsuruoka, Y. Liu, T. Matsuura, and T. Watanabe; "Stability Analysis of Multi-Phase AC Arc Discharge for In-Flight Glass Melting," Current Appl. Phys., 11, S35-S39 (2011).

Tanaka, M., T. Ikeba, Y. Liu, S. Choi, and T. Watanabe; "Investigation of Electrode Erosion Mechanism of MultiPhase AC Arc by High-Speed Video Camera," J. Phys.: Conf. Seri., 441, 012015 (2013)

Tanaka, M., T. Hashizume, T. Imatsuji, Y. Nawata, and T. Watanabe; "Investigation of Erosion Mechanism of Tungsten-Based Electrode in Multi-Phase AC Arc by High-Speed Visualization of Electrode Phenomena," Jpn. J. Appl. Phys., 55, 07LC01 (2016)

Tanaka, M., T. Hashizume, K. Saga, T. Matsuura, and T. Watanabe; "Diode-Rectified Multiphase AC Arc for Improvement of Electrode Erosion Characteristics," $J$. Phys., D: Appl. Phys., 50, 465604 (2017)

Watanabe, T., K. Yatsuda, Y. Yao, T. Yano, and T. Matsuura; "Innovative In-Flight Glass Melting Technology using Thermal Plasmas," Pure and Appl. Chem., 82, 1337-1351 (2010). 